\title{
Subscriber Location in CDMA Cellular Networks
}

\author{
James Caffery, Jr., Student Member, IEEE, and Gordon L. Stüber, Senior Member, IEEE
}

\begin{abstract}
Subscriber radio location techniques are investigated for code-division multiple-access (CDMA) cellular networks. Two methods are considered for radio location: measured times of arrival (ToA) and angles of arrival (AoA). The ToA measurements are obtained from the code tracking loop in the CDMA receiver, and the AoA measurements at a base station (BS) are assumed to be made with an antenna array. The performance of the two methods is evaluated for both ranging and two-dimensional (2-D) location, while varying the propagation conditions and the number of BS's used for the location estimate.
\end{abstract}

Index Terms-Cellular CDMA, position location.

\section{INTRODUCTION}

$\mathbf{O}$ VER THE past decade, considerable attention has been given to vehicle location technology, and numerous applications have been proposed. Recently, in a few testbed areas, rental cars outfitted with location devices and map displays have aided visitors in unfamiliar territory [1]. Taxi and delivery drivers have utilized location technology in Tokyo to navigate the myriad of streets. Fleet operators use location technology to improve product delivery times and to improve the efficiency of the fleet management process. Emergency and police dispatchers have also utilized location technology to locate dispatch vehicles and emergencies for improved response times. In cellular telephone networks, location technology could be used for radio resource and mobility management [2], [3]. For example, a service provider who may have multiple agreements with personal communication services (PCS's), cellular, or satellite carriers, could offer its customers the ability to choose a carrier that best suits their needs at a given time and location [4]. Also, the Federal Communication Commission has recently released an order, to be implemented in two phases, requiring cellular service providers to provide a mechanism for generating subscriber location estimates for Enhanced-911 (E-911) services [5]. A further application of location technology is in the rapidly emerging field of intelligent transportation systems (ITS's), which are designed to enhance highway safety, system operating efficiency, environmental quality, and energy utilization in transportation [6], [7]. Each of the above applications requires a method for determining and relaying the location of vehicular and pedestrian mobile stations (MS's).

Manuscript received April 19, 1996; revised March 3, 1997. This work was supported by GTE Mobilnet. Portions of this paper were presented at the 5th IEEE International Symposium on Personal, Indoor, and Mobile Radio Communications (PIMRC), Toronto, Ont., Canada, September 1995.

The authors are with the School of Electrical and Computer Engineering, Georgia Institute of Technology, Atlanta, GA 30332 USA.

Publisher Item Identifier S 0018-9545(98)02477-3.
Automatic vehicle location (AVL) techniques have been studied thoroughly in the literature for the purpose of vehicle location. AVL systems entail the acquisition of information about the location of MS's operating in an area, and all require the processing of that information to form location estimates. There are three basic AVL methods: dead reckoning, proximity systems, and radio location [8]. Dead reckoning computes the direction and distance of travel from a known starting position [8]. In proximity systems, the nearness of an MS to fixed detection devices is used to determine its position. The devices can be anything from magnetic sensors to conventional radio transmitters and receivers.

Radio location systems attempt to locate an MS by measuring the radio signals traveling between the MS and a set of fixed stations (FS's). The signal measurements are first used to determine the length or direction of the radio path, and then the MS position is derived from known geometric relationships [8]. Radio location can be implemented in one of two ways-either the MS transmits a signal which the FS's use to determine its location or the FS's transmit signals that the MS's use to calculate their own positions [e.g., the global positioning system (GPS)]. There are several fundamental approaches for implementing a radio location system including those based on signal-strength [9]-[12], angle of arrival (AoA) [13], and time of arrival (ToA) [3], [14], [15]. It is important to note that line-of-sight (LOS) propagation is necessary for accurate location estimates.

Many of the existing location technologies use dead reckoning, radio location with GPS, or hybrids which require specialized subscriber equipment, the cost of which can severely limit their availability to the average consumer. With these technologies, the MS formulates the location estimate which may be relayed to a central site. Another approach for providing location services is to use the cellular telephone networks. A method has been proposed in [1] which incorporates the cellular network into the location process. However, this service requires a GPS receiver in the MS to determine the location, and the cellular network is only used to relay the location information to a central site. Only one previous work has examined a subscriber location technique that relies solely on the cellular network, that is based on signal attenuation measurements [12].

This paper examines the feasibility and performance of radio location techniques in code-division multiple-access (CDMA) cellular networks. CDMA is the chosen access scheme, since it appears to be the leading candidate for third generation cellular networks. The cellular network is used as the sole means to locate the MS's, and the location estimates are determined through reception of signals that are transmitted by the MS at 
a set of base stations (BS's). This approach has the advantage of requiring no modifications to the subscriber equipment. Specifically, radio location methods based on AoA and ToA are studied. We concern ourselves with performance in terms of absolute accuracy with no concern given to the rate of location updates that can be achieved.

The remainder of this paper is organized as follows. Section II outlines the methods employed for the AoA and ToA techniques that will be used for the performance evaluation. The propagation models for macrocellular and microcellular systems are discussed in Section III, followed by simulation results in Section IV. A discussion of some practical issues for subscriber location is given in Section V, followed by some concluding remarks in Section VI.

\section{RADIO LOCATION SYSTEM}

\section{A. Angle of Arrival}

AoA techniques estimate the location of an MS by using directive antennas or antenna arrays to measure the AoA at several BS's of a signal that is transmitted by the MS [13], [16]. Simple geometric relationships are then used to form the location estimate, based on the AoA measurements and the known positions of the BS's. With the AoA method, a position fix requires a minimum of two BS's in a 2-D plane. In this paper, we consider the error due to multipath propagation, but do not consider angle estimation errors. Multipath propagation, in the form of scattering near and around the MS and BS, will affect the measured AoA. For macrocells, scattering objects are primarily within a small distance of the MS since the BS's are usually located well above surrounding objects [17], [18]. This results in reception of signals from all directions at an MS while the BS receives signals from a small azimuthal spread. For microcells, it has been suggested that the BS's be placed below rooftop level (lamppost height) in order to confine the signal coverage to a small area [18]. As a result, the BS becomes surrounded by local scatterers and signals can arrive at the BS from a much broader range of angles. Consequently, the AoA approach, which may be used for macrocells, is impractical for microcells.

Gans [14] and Jakes [17] have modeled the macrocellular propagation environment as a ring of scatterers about the MS, with the BS well outside the ring. Fig. 1 illustrates this geometry, where the primary scatterers are assumed to be on a ring of radius $a$ about the MS. The distance between the BS and MS, $d$, is assumed to be much greater than $a$. We assume that the MS uses an omnidirectional antenna, so that

$$
p(\gamma)=\frac{1}{2 \pi}, \quad 0 \leq \gamma<2 \pi
$$

The distribution of the AoA at the $\mathrm{BS}, \theta$, is given by

$$
\frac{d \gamma}{d \theta} p(\theta)=2 p(\gamma)
$$

From the geometry of Fig. 1, we find that [14]

$$
d \gamma \cong\left[\left(\frac{a}{d}\right)^{2}-(\beta-\theta)^{2}\right]^{-(1 / 2)} d \theta
$$

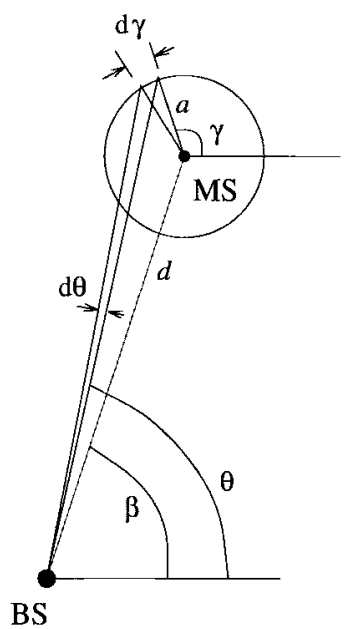

Fig. 1. MS-BS geometry assuming a ring of scatterers for macrocells.

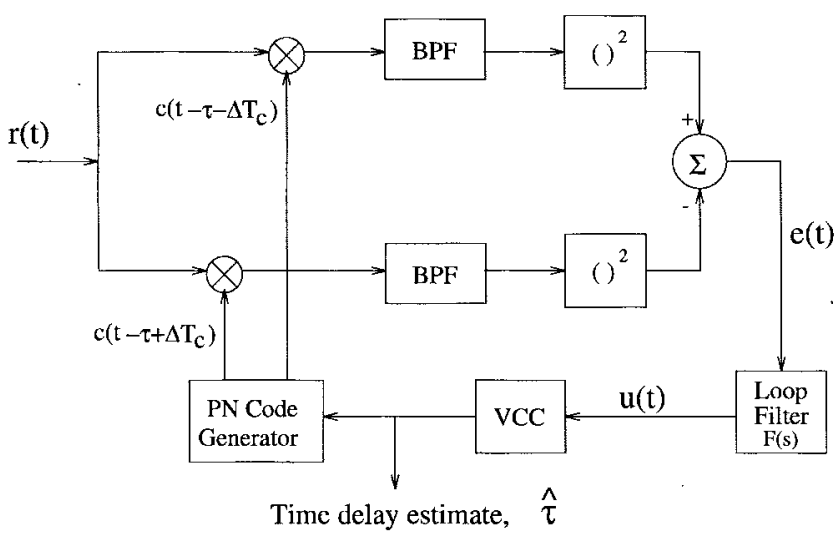

Fig. 2. The DLL used for time-based subscriber location.

Therefore, $p(\theta)$ is

$$
\begin{aligned}
& p(\theta)= \\
& \begin{cases}K\left[\left(\frac{a}{d}\right)^{2}-(\beta-\theta)^{2}\right]^{-(1 / 2)}, & \beta-\theta_{M} \leq \theta \leq \beta+\theta_{M} \\
0, & \text { otherwise }\end{cases}
\end{aligned}
$$

where

$$
\begin{aligned}
\theta_{M} & =\arctan (a / d) \\
K & =\frac{1}{2 \arcsin \left(\frac{d \theta_{M}}{a}\right)} .
\end{aligned}
$$

Note that for $d \gg a$, a small angle approximation can be invoked, with the result that $\theta_{M} \approx a / d$ and $K \approx 1 / \pi$.

The model $p(\theta)$ provides the AoA distribution for signals arriving at a BS. Our model goes one step further by assuming that a measured AoA at a BS also has the distribution $p(\theta)$. Since the measured angles are not equal to the true angles to the MS, the lines of position from the BS's will not intersect at the same point. This problem is resolved by deriving the location estimate from the centroid of the set of points defined by the intersecting lines of position. With three BS's, for example, the lines of position intersect at 
three points: $\left(x_{1}, y_{1}\right),\left(x_{2}, y_{2}\right)$, and $\left(x_{3}, y_{3}\right)$. The location estimate $(\hat{x}, \hat{y})$ is obtained by averaging the coordinates of the points of intersection, i.e., $\hat{x}=\left(x_{1}+x_{2}+x_{3}\right) / 3$ and $\hat{y}=\left(y_{1}+y_{2}+y_{3}\right) / 3$.

\section{B. Time of Arrival}

Many popular radio location techniques are based upon the measurement of the arrival times of a signal transmitted by an MS at several BS's. These methods determine the distance between an MS and BS by measuring the time a signal takes to travel from the BS, to the MS, and back again. Geometrically, this provides a circle, centered at the BS, on which the MS must lie. Using at least three BS's to resolve ambiguities in two dimensions, the intersection of circles provides the MS's position. ${ }^{1}$ This method is often called the ToA method and has the disadvantage that it requires the MS to act as a transponder in which processing delays and non-LOS propagation can introduce error. To overcome these limitations, time difference measurements rather than absolute time measurements can be used. Since the hyperbola is a curve corresponding to a constant time difference of arrival (TDoA) for two BS's, the time differences define hyperbolas, with foci at the BS's, on which the MS must lie. The intersection of hyperbolas provides the location of the MS. This method is often called the TDoA method. Methods for obtaining the ToA or TDoA estimates include phase ranging [19], pulse ranging [3], [19], and spread-spectrum techniques [20], [21].

Since the cellular system being considered is CDMA, methods for determining the ToA's from the spread-spectrum signal are of interest. The two methods for determining time delays in spread-spectrum communications systems are coarse timing acquisition with a sliding correlator or matched filter and fine timing acquisition with a delay-locked loop (DLL) or taudither loop (TDL) [22]. Previous subscriber location studies have used coarse timing acquisition to obtain the ToA estimates [20], [21]. Since the DLL finely tracks the time delay, it is better suited for a location system. The DLL is an essential part of time estimation used for GPS and provides reasonable accuracy over the satellite-earth propagation channel. Here, the DLL-based location system will be investigated for its performance in cellular propagation environments.

The DLL shown in Fig. 2 allows fine synchronization of the local spreading code with the incoming code. It operates by correlating the received signal with the early and late spreading codes $c\left(t-\hat{\tau}+\Delta T_{c}\right)$ and $c\left(t-\hat{\tau}-\Delta T_{c}\right)$, respectively, where $\hat{\tau}$ is an estimate of the delay between the local and incoming codes. The code phase error signal $e(t)$ is obtained by squaring and differencing the correlator outputs. The squaring operations serve to remove the effects of data modulation and carrier phase shift. The loop is closed by applying $e(t)$ to a low-pass filter, whose output is used to drive the voltagecontrolled clock (VCC) and correct the code phase error of the locally generated code. The parameter $\Delta, 0<\Delta<1$, is called the early-late discriminator offset. The output of the VCC provides the ToA estimate $\hat{\tau}$.

\footnotetext{
${ }^{1}$ In general, locating an MS in $n$ dimensions requires $n+1$ measurements
}

\section{Time-Based Location Algorithm}

Two approaches are generally used to calculate the location of an MS from ToA or TDoA estimates. One approach uses a geometric interpretation to calculate the intersection of circles or hyperbolas, depending on whether ToA or TDoA is used. This approach becomes difficult if the hyperbolas or circles do not intersect at a point due to time measurement errors. A second approach calculates the position using a nonlinear least-squares (NL-LS) solution [3], [19], [23], which is a more statistically justifiable approach. The algorithm assumes that the MS, located at $\left(x_{0}, y_{0}\right)$, transmits its sequence at time $\tau_{0}$. The $N$ BS receivers located at coordinates $\left(x_{1}, y_{1}\right)$, $\left(x_{2}, y_{2}\right), \cdots$, and $\left(x_{N}, y_{N}\right)$ receive the sequence at times $\tau_{1}, \tau_{2}, \cdots, \tau_{N}$. As a performance measure, we consider the function [19]

$$
f_{i}(\mathbf{x})=c\left(\tau_{i}-\tau\right)-\sqrt{\left(x_{i}-x\right)^{2}+\left(y_{i}-y\right)^{2}}
$$

where $c$ is the speed of light, and $\mathbf{x}=(x, y, \tau)^{T}$. This function is formed for each BS receiver, $i=1, \cdots, N$, and all the $f_{i}(\mathrm{x})$ could be made zero with the proper choice of $x, y$, and $\tau$. However, the measured values of the arrival times $\tau_{i}$ are generally in error due to multipath and other impairments, and non-LOS propagation introduces errors into the range estimates that are derived from the arrival times.

1) Unconstrained NL-LS Approach: To obtain the location estimate from the raw ToA data, the following function is formed:

$$
F(\mathrm{x})=\sum_{i=1}^{N} \alpha_{i}^{2} f_{i}^{2}(\mathbf{x})
$$

where the $\alpha_{i}$ 's are weights reflecting the reliability of the signal received at BS $i$. The location estimate is determined by minimizing the function $F(\mathrm{x})$.

A simple approach for solving the nonlinear least squares problem in (6) is the steepest descent method, where successive location estimates are updated according to the recursion

$$
\mathrm{x}_{k+1}=\mathrm{x}_{k}-\mu \nabla_{\mathbf{x}} F\left(\mathbf{x}_{k}\right)
$$

where $\mu$ is a constant (scalar or diagonal matrix), $\mathrm{x}_{k}=$ $\left(x_{k}, y_{k}, \tau_{k}\right)^{T}, \nabla_{\mathbf{x}} \equiv d / d \mathbf{x}$, and

$$
\begin{aligned}
\nabla_{\mathbf{x}} F\left(\mathbf{x}_{k}\right) & \left.\equiv \nabla_{\mathbf{x}} F(\mathbf{x})\right|_{\mathbf{x}_{k}}=\left(\begin{array}{l}
\left.\frac{\delta F}{\delta x}\right|_{x_{k}} \\
\left.\frac{\delta F}{\delta y}\right|_{y_{k}} \\
\left.\frac{\delta F}{\delta \tau}\right|_{\tau_{k}}
\end{array}\right) \\
& =\left[\begin{array}{c}
2 \sum_{i=1}^{N} \alpha_{i}^{2} f_{i}\left(\mathbf{x}_{k}\right) \frac{x_{i}-x_{k}}{\sqrt{\left(x_{i}-x_{k}\right)^{2}+\left(y_{i}-y_{k}\right)^{2}}} \\
2 \sum_{i=1}^{N} \alpha_{i}^{2} f_{i}\left(\mathbf{x}_{k}\right) \frac{y_{i}-y_{k}}{\sqrt{\left(x_{i}-x_{k}\right)^{2}+\left(y_{i}-y_{k}\right)^{2}}} \\
-2 c \sum_{i=1}^{N} f_{i}\left(\mathbf{x}_{k}\right)
\end{array}\right] .
\end{aligned}
$$


Since $\tau$ is small (microseconds) compared to $x$ and $y$ (meters), the scalar step size $\mu$ should be small enough to allow $\tau$ to converge to a solution. Consequently, $\mu$ is chosen to be the diagonal matrix

$$
\mu=\left[\begin{array}{ccc}
\mu_{x} & 0 & 0 \\
0 & \mu_{y} & 0 \\
0 & 0 & \mu_{\tau}
\end{array}\right]
$$

where $\mu_{x}, \mu_{y} \gg \mu_{\tau}$. The recursion in (7) continues until $\left\|\nabla_{\mathbf{x}} F\left(\mathbf{x}_{k}\right)\right\|$ is smaller than some prescribed tolerance $\epsilon$.

One drawback of the steepest descent method is its slow convergence. Other algorithms have been investigated [19], [23], which form the solution to (6) by linearizing $f_{i}(\mathbf{x})$ with a Taylor series expansion about $\mathrm{x}_{k}$ and keeping only the first order terms, i.e.,

$$
f_{i}(\mathbf{x}) \approx f_{i}\left(\mathrm{x}_{k}\right)+\delta^{T} \nabla_{\mathbf{x}} f_{i}\left(\mathrm{x}_{k}\right)
$$

where $\boldsymbol{\delta}=\left(\delta_{x}, \delta_{y}, \delta_{\tau}\right)^{T}=\mathrm{x}-\mathrm{x}_{k}$. Substituting (11) into (6) and solving

$$
\nabla_{\boldsymbol{\delta}} F(\mathbf{x})=\mathbf{0}
$$

for $\delta$, the vector $\mathrm{x}_{k}$ is updated by

$$
\mathrm{x}_{k+1}=\mathrm{x}_{k}+\boldsymbol{\delta} .
$$

This new estimate is substituted back into (11) and the process is reiterated until $\left|\delta_{x}\right|+\left|\delta_{y}\right|+c\left|\delta_{\tau}\right|<\epsilon$, where $\epsilon$ is a prescribed tolerance.

When the MS is either close to the BS's or near the perimeter of the area defined by the polygon with the BS's as its vertices, then the linear approximation approach has convergence problems [3], [19]. For microcells, the MS is always within a short distance of the serving BS, so this method is not appropriate. The convergence problem arises from the approximation of $f_{i}(\mathrm{x})$ with the linear terms of the Taylor series expansion. Other objective functions $F(\mathbf{x})$ can be formed replacing, for example, $f_{i}^{2}(\mathrm{x})$ with $\left|f_{i}(\mathrm{x})\right|$. However, these methods usually do not perform as well as minimizing the sum of squares [3].

2) Constrained NL-LS Approach: It may be possible to improve the time-based location algorithm due to the fact that the range error is always positive [24]. This is because the ToA estimates are always greater than the true ToA values due to multipath propagation and other impairments. Also, the range estimates derived from the ToA estimates are greater than the true ranges due to non-LOS propagation. Therefore, the true location of the MS must lie inside the circles of radius $r_{i}=c\left(\tau_{i}-\tau\right), i=1, \cdots, N$, about the $N$ BS's, since the MS cannot lie farther from a BS than its corresponding range estimate (Fig. 3). Mathematically, this implies

$$
r_{i}=c\left(\tau_{i}-\tau\right) \geq \sqrt{\left(x_{i}-x\right)^{2}+\left(y_{i}-y\right)^{2}}
$$

where $(x, y)$ is the position of the MS. Since the unconstrained NL-LS algorithm does not take this restriction into account, a constrained NL-LS approach can be used to force the estimate at each iteration to satisfy (14). However, the LS solution is complicated by the nonlinear functionals $f_{i}(\mathbf{x})$ as well as the

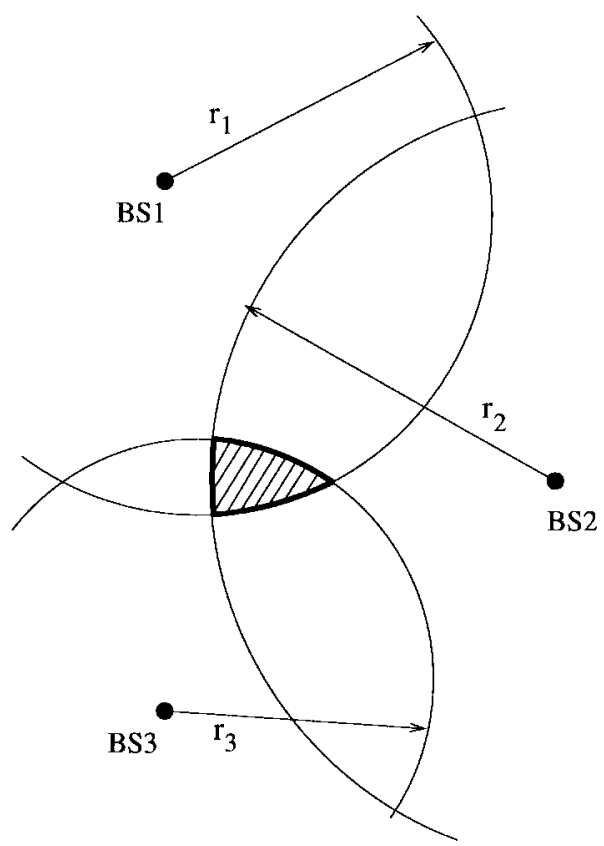

Fig. 3. The location of the MS is constrained to the intersection area (shaded region) of circles of radius $c\left(\tau_{i}-\tau\right)$ centered at each $\mathrm{BS}$

nonlinear inequality constraints of (14). Note that (14) implies that

$$
\sqrt{\left(x_{i}-x\right)^{2}+\left(y_{i}-y\right)^{2}}-c\left(\tau_{i}-\tau\right) \leq 0 .
$$

We recognize from (5) that the left side of the inequality in (15) is simply $g_{i}(\mathbf{x})=-f_{i}(\mathbf{x})$. Hence, the restrictions $f_{i}(\mathbf{x}) \geq 0$ are formed, where the area within the constraint boundaries is known as the feasible region.

There are many approaches to forming numerical solutions for NL-LS problems with nonlinear inequality constraints of the form $g_{i}(\mathbf{x}) \leq 0$ [25]. One simple, yet effective, method uses penalty functions to modify the objective function $F(\mathrm{x})$ and form a solution using an unconstrained approach as in the previous section. The penalty functions provide a large penalty to the objective function when one or more of the constraints are violated. The objective function in (6) is modified to include the penalty functions $g_{i}(\mathbf{x})$ as follows [25]:

$$
F(\mathbf{x})=\sum_{i=1}^{N} \alpha_{i}^{2} f_{i}^{2}(\mathbf{x})-P \sum_{i=1}^{N}\left[g_{i}(\mathbf{x})\right]^{-1}
$$

where $P$ is positive for minimization. As any constraint is approached during the search, the penalty term forces $F$ toward infinity, thus forming a natural optimum within the feasible region. This approach requires that the initial guess be placed within the feasible region. A method for doing this is described in [25].

The search procedure can be viewed as the optimization of a sequence of surfaces which tend toward the true value of the objective function. Initially, an unconstrained search method is used to provide an artificial optimum $\mathbf{x}_{1}$ with a large value of $P=P_{1}$. The next stage is initialized with the previous estimate $\mathrm{x}_{1}$ and uses a smaller $P=P_{2}$ to provide a better approximation to the true optimum. In this 
TABLE I

COST207 SiX-Tap Reduced Typical Urban Power Delay Profile

\begin{tabular}{cc}
\hline \hline \multicolumn{2}{c}{ COST 207 Model } \\
\hline Delay- $\tau_{0}(\mu \mathrm{s})$ & Fractional Power \\
\hline 0.0 & 0.189 \\
0.2 & 0.379 \\
0.5 & 0.239 \\
1.6 & 0.095 \\
2.3 & 0.061 \\
5.0 & 0.037 \\
\hline \hline
\end{tabular}

way, the solution approaches the constraints more closely, if the optimum happens to lie close to one of the constraints. The penalty constraints become smaller at each stage, forming a monotonic-decreasing sequence $P_{1}>P_{2}>\cdots$, and the sequence of artificial optima $\mathbf{x}_{1}, \mathrm{x}_{2}, \cdots$ tends toward the true optimum. The search continues until several iterations fail to produce a change in the objective function. This formulation essentially replaces a constrained optimization by a sequence of unconstrained optimizations.

\section{Propagation Models}

A three-stage model is used for the radio propagation environment, that includes multipath-fading, shadowing, and path loss. The particular models used in this paper for macrocellular and microcellular propagation environments are now described.

\section{A. Macrocells}

For wideband spread-spectrum systems, the channel can be modeled by the $M$-tap tapped delay line

$$
h(t)=\sum_{i=0}^{M} w_{i}(t) \delta\left(t-\tau_{i}\right)
$$

where the $\left\{\tau_{i}\right\}$ are the tap delays and the $\left\{w_{i}\right\}$ are the tap gains, assumed here to be complex Gaussian random processes. For numerical convenience, the tap delays can be chosen to be an integer multiple of some small delay $\tau$, i.e., $\tau_{i}=k_{i} \tau, i=1, \cdots, M$. The first tap delay $\tau_{0}$ is determined from the MS-BS geometry of Fig. 1 by calculating the distance traveled by a signal transmitted from the MS in a random direction according to $p(\gamma)$ and reflected from the ring of scatterers to the BS. The remaining delays are chosen according to the six-tap reduced typical urban delay profile defined in COST207 [26] (see Table I). The model deviates slightly from the COST207 model by assuming a classical Doppler spectrum for all taps, i.e., in the simulations the taps gains are all generated by using Jakes' method [17].

Shadow fades have been described from measurements as being lognormally distributed with a standard deviation that depends on the frequency and the environment [18]. Gudmundson [27] has suggested a simple Markovian model to describe variations in the local mean envelope (or squared envelope) level due to shadow variations. With this model

$$
\Omega_{k+1(\mathrm{~dB})}=\xi \Omega_{k(\mathrm{~dB})}+(1-\xi) \eta_{k}
$$

where $\Omega_{k(\mathrm{~dB})}$ is the local mean envelope (or square envelope) level (in decibels) that is experienced at location $k, \xi$ is a parameter that controls the spatial decorrelation of the shadowing, and $\left\{\eta_{k}\right\}$ is a zero-mean discrete-time Gaussian random process with autocorrelation $\phi_{\eta \eta}(n)=\tilde{\sigma}^{2} \delta(n)$. The autocorrelation of $\Omega_{k(\mathrm{~dB})}$ is given by

$$
\phi_{\Omega_{(\mathrm{dB})} \Omega_{(\mathrm{dB})}}(n)=\frac{1-\xi}{1+\xi} \tilde{\sigma}^{2} \xi^{|n|}=\sigma_{s}^{2} \xi^{|n|}
$$

where $\sigma_{s}$ is called the shadow standard deviation. Typical values of the shadow standard deviation range from 5 to 12 $\mathrm{dB}$ in macrocells [17], [18], [28]. If we assume that the local mean is sampled every $T \mathrm{~s}$, then the autocorrelation can be expressed as

$$
\phi_{\Omega_{(\mathrm{dB})} \Omega_{(\mathrm{dB})}}(k) \equiv \phi_{\Omega_{(\mathrm{dB})} \Omega_{(\mathrm{dB})}}(n=k T)=\sigma_{s}^{2} \epsilon_{D}^{(v T / D)|k|}
$$

where $\epsilon_{D}$ determines the correlation between two points separated by a spatial distance $D$ and $v$ is the velocity of the MS. The simulations in the sequel assume a shadow decorrelation of 0.1 at a distance of $30 \mathrm{~m}$.

Several empirical path loss models have been presented in the literature, one of the most useful being Hata's model [28], which expresses the path loss in terms of the carrier frequency, BS height, MS antenna height, and the type of environment (urban, suburban, or rural). Hata's model for medium or small city urban areas is used in the sequel with a carrier frequency of $f=850 \mathrm{MHz}$, BS antenna height of $100 \mathrm{~m}$, and an MS antenna height of $2.5 \mathrm{~m}$.

\section{B. Microcells}

The wideband channel and shadowing models discussed above can also be used to model microcellular propagation. However, the power delay profiles are different, and the standard deviation of shadowing in microcells typically ranges from 4 to $13 \mathrm{~dB}$. Further differences in the propagation models for microcells and macrocells are discussed in the following.

Microcellular path loss is often described by a two-slope characteristic, where the area mean $\bar{\Omega}=\mathrm{E}[\Omega]$ is given by [29]

$$
\bar{\Omega}=10 \log _{10}\left[\frac{A}{d^{a}(1+d / g)^{b}}\right] \mathrm{dBm}
$$

where $A$ is a constant, $d$ is the radio path length, $g$ is the break point (that ranges from 150 to $300 \mathrm{~m}$ ), and $a$ and $b$ determine the slopes before and after the break point. In the simulations, we assume $g=150 \mathrm{~m}$ and $a=b=2$.

An important consideration for microcells is the corner effect, which occurs in microcellular scenarios when an MS rounds a street corner. To account for this effect, LOS propagation is assumed to the MS until it rounds the corner. The non-LOS propagation after rounding a street corner is modeled by assuming LOS propagation from an imaginary transmitter that is located at the street corner having a transmit power equal to the received power at the street corner from the serving BS. The area mean (in $\mathrm{dBm}$ ) is given by (22), at the bottom of the next page, where $d_{c}$ is the distance between the serving $\mathrm{BS}$ and the corner. 


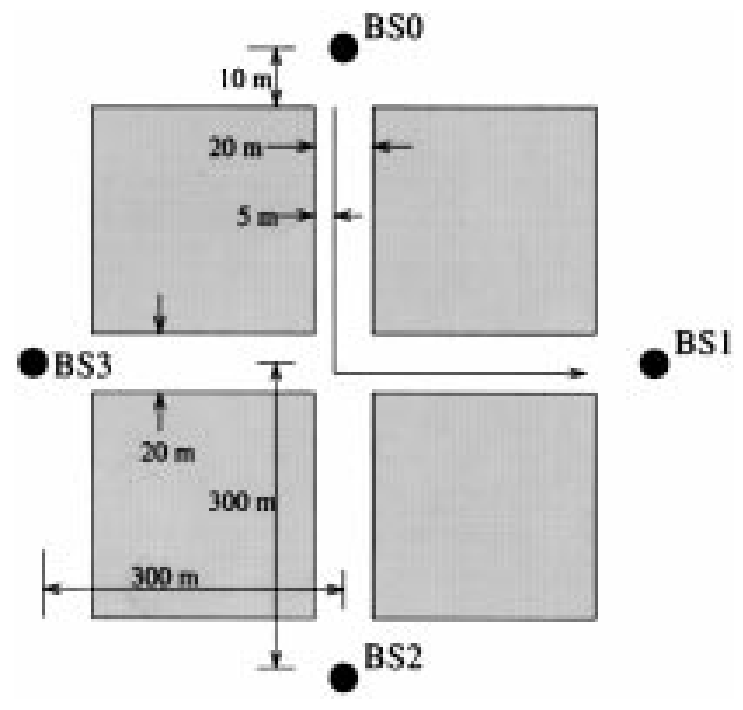

Fig. 4. Manhattan street microcell deployment.

Due to the site-specific nature of the microcellular propagation environment, techniques such as ray tracing have been developed. In this study, ray tracing concepts are used to calculate the propagation delays for the wideband channel model. A Manhattan street microcell BS deployment is assumed as shown in Fig. 4. When the MS is LOS with a BS, a four-path model is used, consisting of a direct path, a roadreflected path, and two wall-reflected paths. The taps of the wideband channel model are generated using Jakes' method, appropriately modified for Rician fading. When the MS is non LOS with a BS, i.e., around the corner, a different approach is taken to determine the propagation delays. Since the literature provides no results that describe the power delay profile for an MS that is around a corner from a BS, a simplistic model is chosen. A four-path non-LOS propagation model is used that includes two paths that arrive from diffractions at the building corners in the street intersection and two remaining paths whose delays are generated by adding random delays to the first two paths. All paths are assumed to be Rayleigh faded. The model chosen here is inconsequential, because the extra time delay for non-LOS BS's introduces a large amount of error into the location algorithm. Hence, accurate modeling of multipath propagation on non-LOS streets is not necessary; only a means of introducing the excess propagation delay around the street corner is needed.

\section{SimULATIONS}

The location techniques described in Section II were simulated in the macrocellular and microcellular environments described in Section III to determine their performance. The spreading code used was an $m$ sequence of length 127 and chip rate $T_{c}^{-1}=1.2288 \mathrm{Mcps}$. In the DLL, an all-pass filter was used for the loop filter [i.e., $F(s)=1$ ]. For the VCC, the output time delay estimate and input waveform are related by

$$
\hat{\tau}(t)=K_{\mathrm{VCC}} T_{c} \int_{0}^{t} u(x) d x
$$

where $K_{\mathrm{VCC}}$ is the gain of the $\mathrm{VCC}, T_{c}$ is the chip period, $u(t)$ is the output of the loop filter, and the VCC is assumed to begin operating at time $t=0$. A simple accumulator models the operation of the VCC in the computer simulations with the constant $K_{\mathrm{VCC}} T_{c}=0.003$. Note that there is a limitation in the accuracy that can be achieved when simulating the DLL on a computer. As a result, we limit the resolution of the DLL to $1 / 120$ of a chip to limit the simulation time. Consequently, the ranging resolution is limited to approximately $2 \mathrm{~m}$, which causes all range estimates to be in error even in the absence of propagation impairments. However, with such a fine resolution, propagation impairments will be the predominant source of location error.

\section{A. Range Estimation}

Ranging measures the 1-D distance between an MS and BS. Only the time-based method is employed for ranging since AoA ranging does not make sense. For macrocells, our ranging results assume that the first path to arrive from the COST 207 model is a LOS path. Consequently, the ranging results for macrocells are very optimistic by disregarding the extra propagation caused by non-LOS propagation when a direct path does not exist. For microcells, the Manhattan street microcell deployment in Fig. 4 is assumed.

1) Effect of Standard Deviation of Shadowing, $\sigma_{s}$ : Fig. 5(a) shows the effect of the shadow standard deviation on the mean and standard deviation of the range estimation error with an early-late discriminator offset $\Delta=1 / 2$ and a chip-energy-tonoise ratio $E_{c} / N_{o}=10 \mathrm{~dB}$. The mean ranging error increases by approximately $10 \mathrm{~m}$ as $\sigma_{s}$ increases from 4 to $12 \mathrm{~dB}$. The standard deviation of the ranging error also increases due to the increased variability of the shadowing process.

2) Effect of $\mathbf{E}_{\mathbf{c}} / \mathbf{N}_{\mathbf{o}}$ : Fig. 5(b) shows the effect of $E_{c} / N_{o}$ on the ranging error with $\Delta=1 / 2$ and $\sigma_{s}=6 \mathrm{~dB}$. The increase in ranging error for decreasing $E_{c} / N_{o}$ is expected in any system. The effect is not as pronounced in the microcellular environment due to the smaller delay spreads.

3) Effect of $\boldsymbol{\Delta}$ : The effect of multipath on the tracking ability of the DLL can be explained by observing the distortion that multipath causes on the correlation function of the spreading code, which has a triangular shape for a rectangular chip-shaping pulse. Fig. 6 shows an example of a distorted loop $\mathrm{S}$ curve for the case of two multipath components, the second having half the power of the first and delayed by $T_{c} / 2$. Observe that the tracking error introduced by multipath propagation is reduced by using smaller $\Delta$. However, the

$$
\bar{\Omega} \sim \begin{cases}10 \log _{10}\left[\frac{1}{d^{a}(1+d / g)^{b}}\right], & d \leq d_{c} \\ 10 \log _{10}\left\{\frac{1}{d_{c}^{a}\left(1+d_{c} / g\right)^{b}} \cdot \frac{1}{\left(d-d_{c}\right)^{a}\left[1+\left(d-d_{c}\right) / g\right]^{b}}\right\}, & d>d_{c}\end{cases}
$$




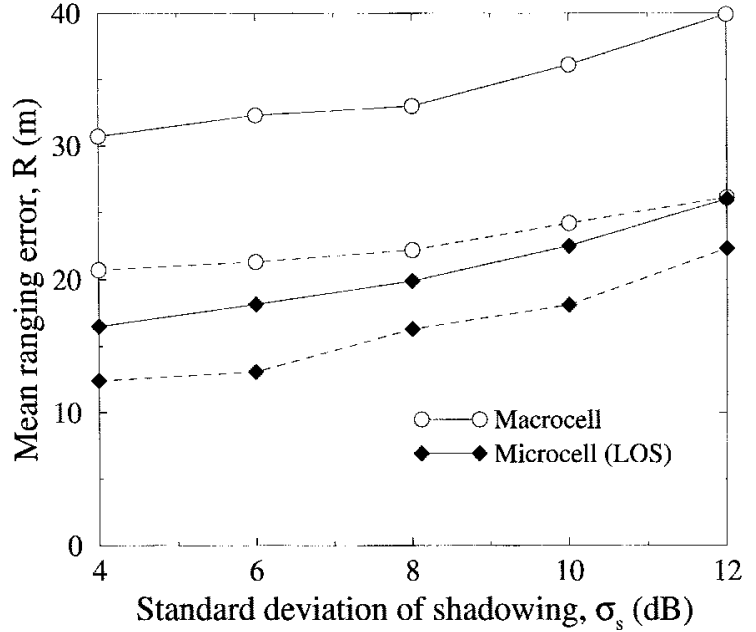

(a)

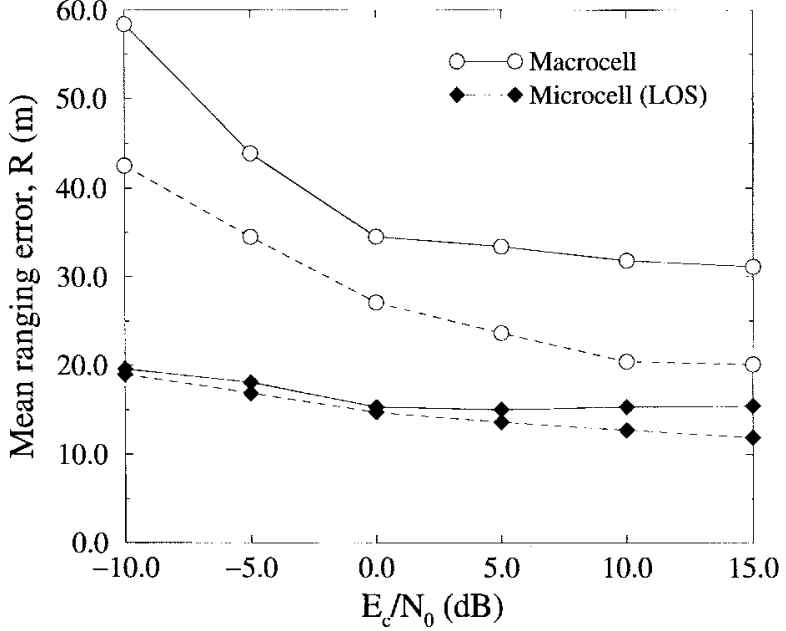

(b)

Fig. 5. Effect of (a) shadow standard deviation $\left(E_{c} / N_{o}=10 \mathrm{~dB}\right)$ and (b) $E_{c} / N_{o}\left(\sigma_{s}=6 \mathrm{~dB}\right)$ on the mean (solid lines) and standard deviation (dashed lines) of the ranging error.

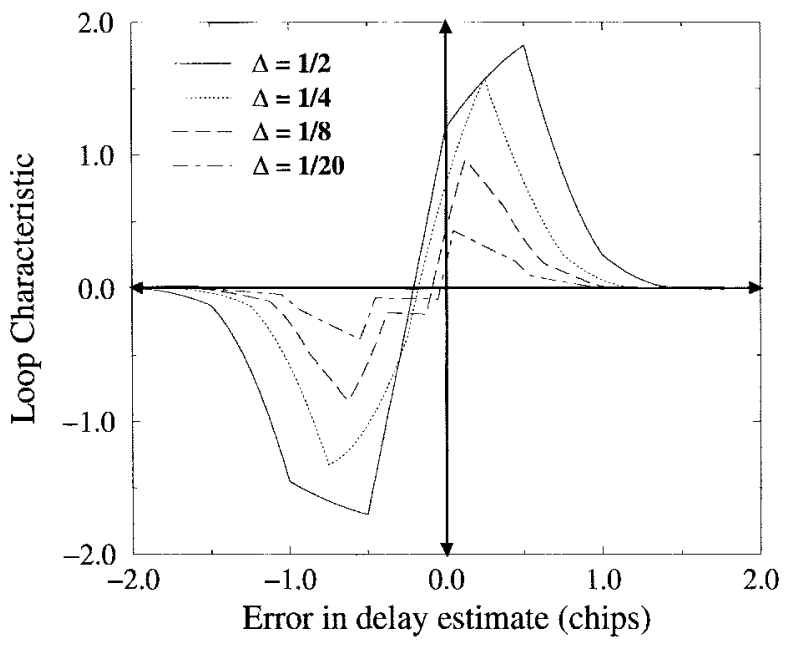

Fig. 6. Distortion of the $\mathrm{S}$ curve due to multipath for different values of $\Delta$.

minimum size of $\Delta$ is limited by hardware considerations (such as the clock rate) and the precorrelation bandwidth in the DLL. Band limiting tends to round the autocorrelation peak which limits the discrimination between the early and late correlation when using small $\Delta$ [30].

Simulation results for various $\Delta$ are presented in Table II with $\sigma_{s}=6 \mathrm{~dB}$ and $E_{c} / N_{o}=5 \mathrm{~dB}$. The results show that the ranging error mean and standard deviation can be significantly reduced by using a smaller $\Delta$.

\section{B. Two-Dimensional Location}

Two-dimensional (2-D) location estimates the MS location by using several BS's. Here, we focus on the accuracy of the location estimates as a function of the number of BS's. This is an important consideration, since using more BS's means more processing and an increased load on the network. Assuming a transmit power of $1 \mathrm{dBW}$ (the maximum for Class III IS$95 \mathrm{MS}$ 's), a noise power of $-100 \mathrm{dBm}$ was added to each BS receiver for the time-based method. The macrocell and microcell deployment scenarios are as follows.
TABLE II

Mean Ranging Error and Standard Deviation for Various Values of $\Delta$. Values Are in Meters

\begin{tabular}{ccccc}
\cline { 2 - 5 } & \multicolumn{2}{c}{ Microcell } & \multicolumn{2}{c}{ Macrocell } \\
\hline$\Delta$ & Mean & Std. Dev & Mean & Std. Dev \\
\hline $1 / 2$ & 15.6 & 15.5 & 30.8 & 23.6 \\
\hline $1 / 4$ & 10.8 & 12.1 & 25.3 & 19.1 \\
\hline $1 / 8$ & 4.5 & 6.1 & 16.9 & 18.9 \\
\hline \hline
\end{tabular}

1) Macrocells: We assume a distance of $6000 \mathrm{~m}$ between BS's, i.e., the cell radius is $3000 \mathrm{~m}$. Assuming known BS positions, the MS is randomly placed among the BS's and the nearest BS's are used for the location process. For macrocells, ToA and AoA approaches are compared when using two-five BS's in the location process as a function of the scattering radius $a$ about the MS.

The simulations examined both the unconstrained and constrained location algorithms of Section II-C, which almost always converged with $\alpha<1$ for each BS. The mean and standard deviation of the location error for the ToA method using the unconstrained NL-LS algorithm are shown in Fig. 7(a). For a given scattering radius, the mean and standard deviation decreases when more BS's are used. As expected, a larger scattering radius increases the location error due to nonLOS propagation. Recall that LOS propagation is necessary for accurate ranging and location estimates.

The mean and standard deviation of the location error for the ToA method with the constrained NL-LS algorithm are shown in Fig. 7(b). Unlike the unconstrained NL-LS case, the performance is not improved significantly when more BS's are used. Table III compares the performance of the unconstrained and constrained NL-LS methods, where both algorithms are initialized with the same location estimate. The mean location error is reduced up to $30 \%$ by using the constrained NL-LS algorithm. The constrained NL-LS with three BS's performs nearly as well as the unconstrained NL-LS with five BS's. This implies that the constrained NL-LS algorithm can result in less network loading. 


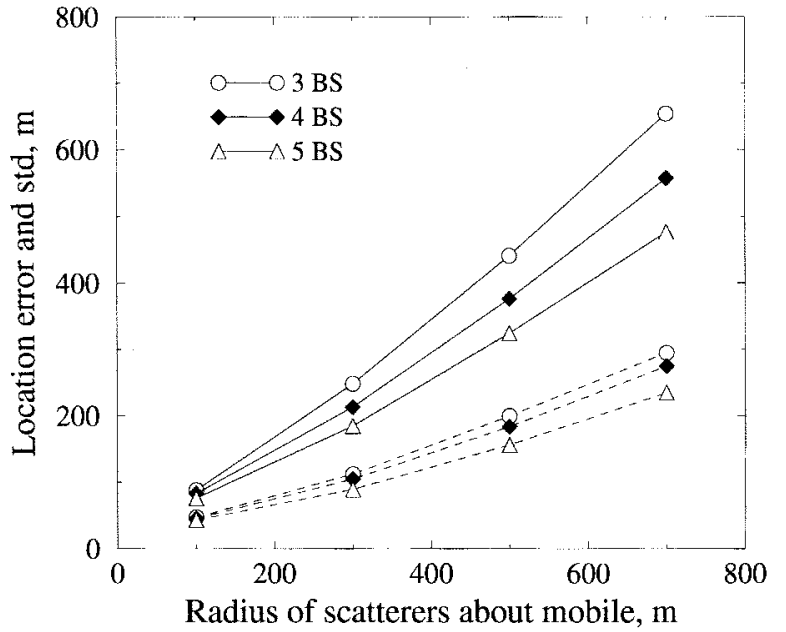

(a)

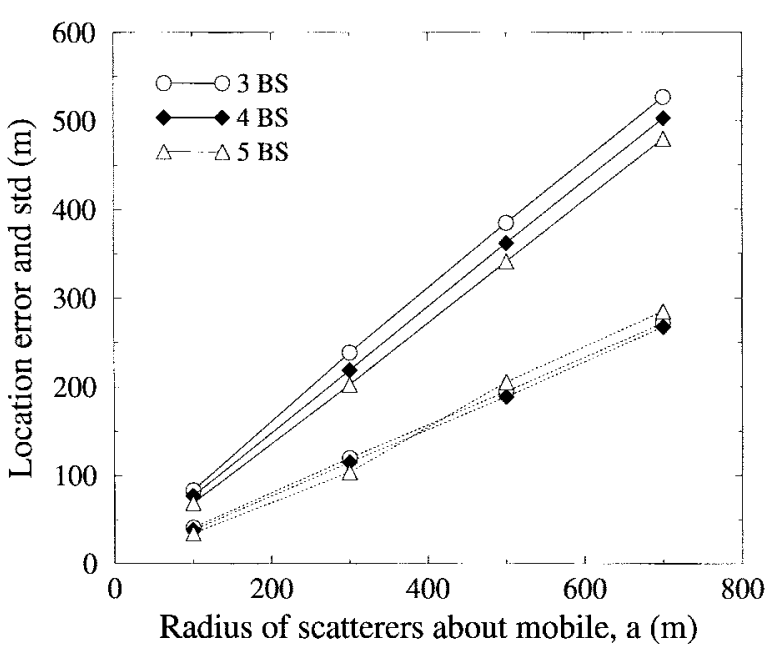

(b)

Fig. 7. Two-dimensional location in macrocells for the ToA method using the (a) unconstrained and (b) constrained NL-LS algorithms. Solid lines denote the mean error, and dashed lines denote the standard deviation: $\sigma_{s}=6 \mathrm{~dB}$.

TABLE III

Reduction in the Mean Location ERRor of the Constrained NL-LS Algorithm Compared to the UnConstrained NL-LS Algorithm for the TOA Method in Macrocells. Values Are in Meters

\begin{tabular}{c|cccc}
\hline \hline \# BSs & $a=100$ & $a=300$ & $a=500$ & $a=700$ \\
\hline 3 & 10.0 & 50.5 & 125.7 & 213.2 \\
4 & 4.8 & 29.0 & 75.7 & 145.0 \\
5 & 6.1 & 23.1 & 53.4 & 98.8 \\
\hline \hline
\end{tabular}

Results for the AoA method are shown in Fig. 8. Once again, a larger number of BS's decreases the mean and standard deviation of the location error. However, diminishing returns are obtained by increasing the number of BS's. For $a=100 \mathrm{~m}$ and $a=300 \mathrm{~m}$, the unconstrained ToA method outperforms the AoA method for the same number of BS's. For larger scattering radii, the AoA method steadily improves and performs slightly better than the unconstrained ToA method at a radius of $a=700 \mathrm{~m}$ for the same number of BS's. In all cases, the constrained ToA method performs the best.

2) Microcells: As the MS rounds the corner from BS0 to BS1 in Fig. 4, its location is estimated using a combination of BS's. For this particular deployment, we assume LOS propagation between the MS and two BS's (four at an intersection) and non-LOS propagation to the other two BS's. Only the ToA method is used since the AoA method is unreliable due to the relatively large AoA spreads in microcells. The number of BS's used to derive the location estimate ranges from two to four. With 2-BS location, BS0 and BS2 are used until the MS rounds the corner after which BS1 and BS3 are used. With 3BS location, BS0, BS1, and BS2 are used until the MS rounds the corner after which BS0, BS1, and BS3 are used.

Fig. 9 plots the results for the microcell deployment in Fig. 4, and indicates that the location accuracy is improved with more BS's. Fig. 9 shows the effect of the NL-LS weighting factors $\alpha$ on the location performance. The LOS BS's weights are $\alpha=1$, whereas the non-LOS BS weights were varied from 0.2 to 1.0. Fig. 9(a) shows that a smaller $\alpha$ can significantly reduce the mean and standard deviation of the location

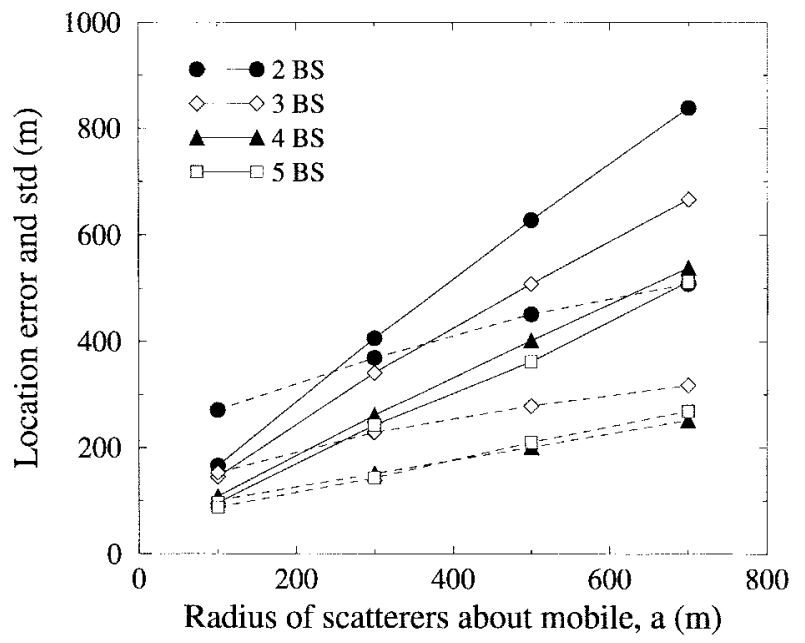

Fig. 8. Two-dimensional location in macrocells using the AoA method. Solid lines denote the mean error, and dashed lines denote the standard deviation.

error with the unconstrained NL-LS algorithm, especially for 3 -BS location. For the constrained NL-LS algorithm, there is no significant improvement for $\alpha<0.6$, as shown in Fig. 9(b). It is interesting to note that 4-BS location is much better than 3-BS location, even though an additional non-LOS BS is used. This is because the two non-LOS BS's tend to "cancel" one another's effects as a result of the symmetry of the BS layout.

Two-BS location was also considered, using the two LOS BS's with $\alpha=1$ for both BS. The mean location error was 6.9 $\mathrm{m}$ with a standard deviation of $2.0 \mathrm{~m}$ for both the unconstrained and constrained NL-LS algorithms. Although three BS's are required for 2-D location, the constraint that the MS must lie on a line between the two LOS BS's provides the additional information needed for 2-BS location. This topographical constraint provides more accurate location information than the additional information from a non-LOS ToA measurement and, therefore, 2-BS location performs better than 3- or 4-BS location. Note that the feasibility of this approach depends on the BS topography. 


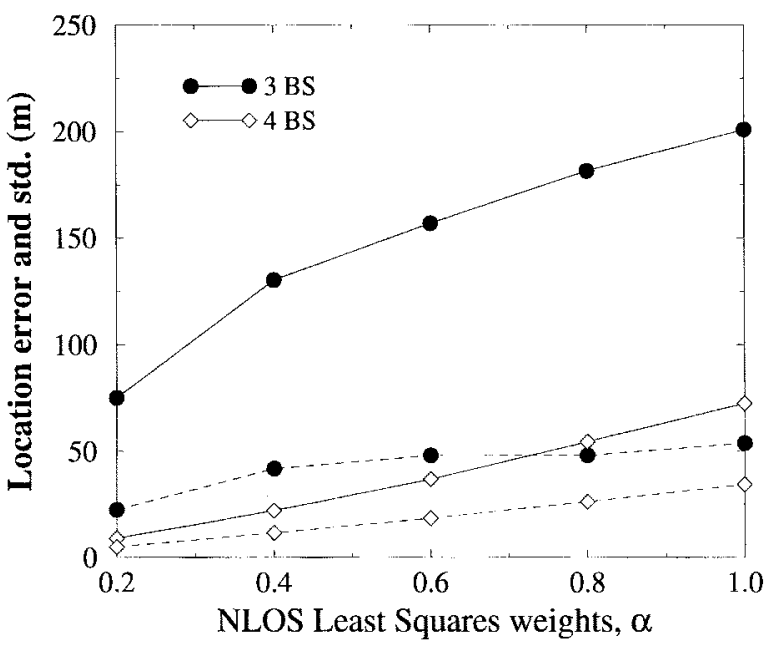

(a)

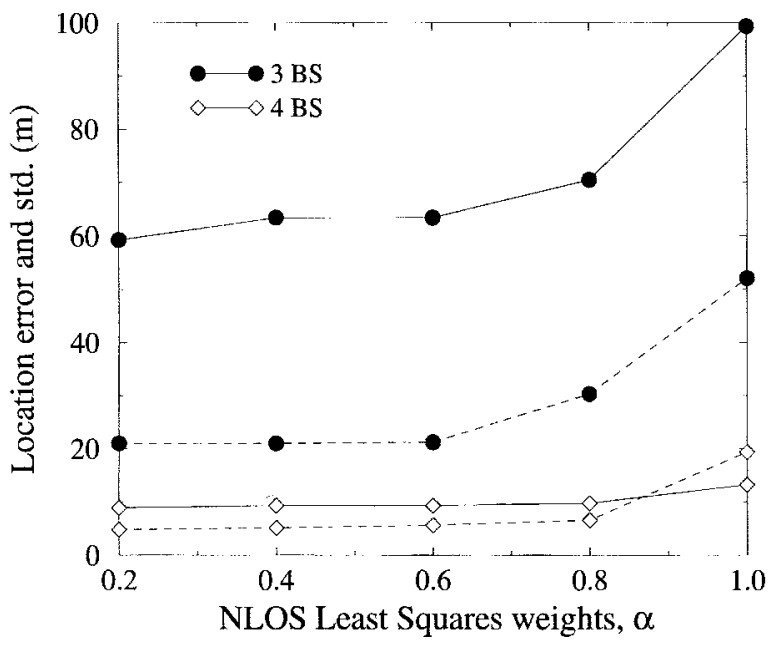

(b)

Fig. 9. Two-dimensional location in microcells using the (a) unconstrained and (b) constrained NL-LS algorithms for various values of the non-LOS BS weights, $\alpha$. Solid lines denote the mean error, and dashed lines denote the standard deviation.

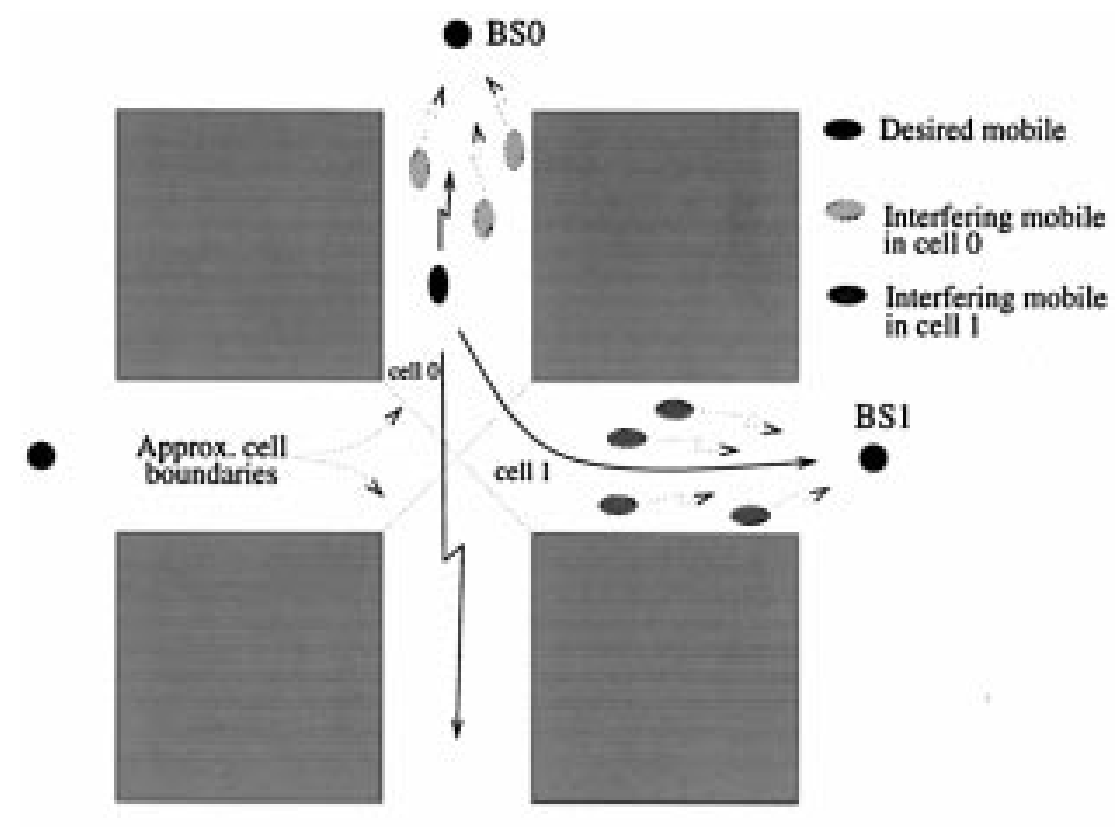

BS2

Fig. 10. CDMA multiuser interference scenario.

\section{DISCUSSION}

The implementation of an effective cellular radio location system, requires a method for identifying the set of BS's to be used for deriving the location estimate. This is especially important for microcells since the use of non-LOS BS's can introduce large errors in the location estimates as it does in macrocells. The link quality measurements used for handoff initiation may be used to determine the appropriate set of BS's. The appropriate BS's can be determined, for example, by received signal strength measurements.

In order to achieve high accuracy, LOS propagation is necessary between the MS and BS's used in the location process. This may be impossible to achieve in macrocells, but may be attainable in microcells. The additional propagation time to a non-LOS BS leads to a positive range estimate error. Recently, a statistical method has been suggested to compensate for non-LOS induced errors by biasing the range estimates [31].

This paper has not addressed the effect of CDMA multiuser interference caused by the nonorthogonality of the user spreading codes. Multiuser interference along with unequal received power levels from different users leads to the near-far effect, which is mitigated in CDMA systems by using power control techniques. This paper has only considered the case of a single 
MS with no interferers. Since the location system requires the use of several BS's, multiuser interference may pose severe problems. For example, consider the microcell deployment in Fig. 10, where the target MS whose location is desired (solid black oval) is being served by BSO. To determine its location, BS0, BS1, and BS2 are used. If power control is used, then the signals from all MS's served by BS0 (black and light grey) will arrive at BS0 with approximately the same power. The same is true for the MS's being served by BS1 (medium grey) and BS2 (not shown). To derive the location estimate, BS1 and BS2 must detect the signal being transmitted by the target MS (black oval). However, the signal from the target MS may experience severe multiuser interference from MS's being served by BS1 and BS2, since the target MS is not power controlled by those BS's. This multiuser interference will affect the tracking capability of the code tracking loop that is used to obtain the ToA information.

\section{CONCLUDING REMARKS}

Subscriber location in CDMA cellular networks has been investigated for both macrocellular and microcellular deployments. For range estimation, it was seen that location error and standard deviation increases with increasing shadow standard deviation and decreasing $E_{c} / N_{o}$. Under the condition of moderate delay spread, the tracking performance of the DLL can be improved by using a smaller early-late discriminator offset $\Delta$. For 2-D location, the location error is reduced by increasing the number of BS's used in the location process. For macrocells, the unconstrained NL-LS ToA method outperforms the AoA method for a small scattering radius, while the AoA method performs slightly better for a large scattering radius. In all cases, the constrained NL-LS ToA method performed best.

It should be noted that our AoA-based location results depend on the method chosen for generating the AoA's, namely, reflection from a ring of scatterers about the MS. A different method for generating the AoA distribution $p(\theta)$ may produce different results. For microcells, the AoA method is inappropriate so only the ToA method was used. Again, using more BS's decreases the error and using small weights for the non-LOS BS's provides good error reduction.

The ToA method relies on an NL-LS solution. This was obtained here by using the steepest descent method, chosen for its simplicity. Better methods for locating the minimum of a least squares surface could be employed, such as the Levenberg-Marquardt method, to improve the convergence time and possibly the accuracy of the location estimates.

\section{REFERENCES}

[1] R. Jurgen, "The electronic motorist," IEEE Spectrum, vol. 32, pp. 37-48, Mar. 1995.

[2] I. Paton, E. Crompton, J. Gardner, and J. Noras, "Terminal self-location in mobile radio systems," in 6th Int. Conf. Mobile Radio and Personal Communications, 1991, pp. 203-207.
[3] H. Hashemi, "Pulse ranging radiolocation technique and its application to channel assignment in digital cellular radio," in IEEE Veh. Technol. Conf., 1991, pp. 675-680.

[4] A. Giordano, M. Chan, and H. Habal, "A novel location-based service and architecture," in IEEE Personal Indoor Mobile Radio Conf., 1995, pp. 853-857.

[5] "Revision of the commissions rules to ensure compatibility with enhanced 911 emergency calling systems, RM-8143," CC Docket 94-102, FCC, Washington, DC, July 26, 1996.

[6] W. Collier and R. Weiland, "Smart cars, smart highways," IEEE Spectrum, vol. 31, pp. 27-33, Apr. 1994.

[7] R. Jurgen, "Smart cars and highways go global," IEEE Spectrum, vol 28, pp. 26-36, May 1991.

[8] S. Riter and J. McCoy, "Vehicle location-An overview," IEEE Trans. Veh. Technol., vol. VT-26, pp. 7-11, Feb. 1977.

[9] W. Figel, N. Shepherd, and W. Trammell, "Vehicle location by a signal attenuation method," IEEE Trans. Veh. Technol., vol. VT-18, pp. 105-110, Nov. 1969.

[10] M. Hata and T. Nagatsu, "Mobile location using signal strength measurements in a cellular system," IEEE Trans. Veh. Technol., vol. VT-29, pp. 245-251, May 1980

[11] G. Ott, "Vehicle location in cellular mobile radio systems," IEEE Trans. Veh. Technol., vol. VT-26, pp. 43-46, Feb. 1977.

[12] H.-L. Song, "Automatic vehicle location in cellular communications systems," IEEE Trans. Veh. Technol., vol. 43, pp. 902-908, Nov. 1994.

[13] S. Sakagami et. al., "Vehicle position estimates by multibeam antennas in multipath environments," IEEE Trans. Veh. Technol., vol. 41, pp. 63-67, Feb. 1992.

[14] M. Gans, "A power-spectral theory of propagation in the mobile-radio environment," IEEE Trans. Veh. Technol., vol. VT-21, pp. 27-38, Feb. 1972.

[15] M. Feuerstein and T. Pratt, "A local area position location system," in 5th Int. Conf. Mobile Radio and Personal Communications, 1989, pp. 79-83.

[16] S. Anderson, M. Millnert, M. Viberg, and B. Wahlberg, "An adaptive array for mobile communication systems," IEEE Trans. Veh. Technol., vol. 40, pp. 230-236, Feb. 1991.

[17] W. C. Jakes, Microwave Mobile Communications. New York: IEEE Press, 1994.

[18] J. Parsons, The Mobile Radio Propagation Channel. New York: Halsted, 1992.

[19] G. Turin, W. Jewell, and T. Johnston, "Simulation of urban vehiclemonitoring systems," IEEE Trans. Veh. Technol., vol. VT-21, pp. 9-16, Feb. 1972.

[20] P. Goud, A. Sesay, and M. Fattouche, "A spread spectrum radiolocation technique and its application to cellular radio," in IEEE Pacific Rim Conf. Communications, Computers and Signal Processing, 1991, pp. 661-664.

[21] J. Caffery, Jr. and G. Stüber, "Vehicle location and tracking for IVHS in CDMA microcells," in IEEE Personal Indoor Mobile Radio Conf., 1994, pp. 1227-1231.

[22] R. Ziemer and R. Peterson, Digital Communications and Spread Spectrum Systems. New York: Macmillan, 1985.

[23] W. Foy, "Position-location solutions by Taylor-series estimation," IEEE Trans. Aerosp. Electron. Syst., vol. AES-12, pp. 187-193, Mar. 1976.

[24] G. Morley and W. Grover, "Improved location estimation with pulseranging in presence of shadowing and multipath excess-delay effects," Electron. Lett., vol. 31, pp. 1609-1610, Aug. 1995.

[25] G. Beveridge and R. Schechter, Optimization: Theory and Practice. New York: McGraw-Hill, 1970.

[26] "Proposal on channel transfer functions to be used in GSM late 1986," COST 207 TD(86)51-REV 3, Sept. 1986.

[27] M. Gudmundson, "Analysis of handover algorithms," in IEEE Veh. Technol. Conf., 1991, pp. 537-541.

[28] G. Stüber, Principles of Mobile Communication. Norwell, MA Kluwer, 1996.

[29] P. Harley, "Short distance attenuation measurements at $900 \mathrm{MHz}$ and $1.8 \mathrm{GHz}$ using low antenna heights for microcells," IEEE J. Select. Areas Commun., vol. 7, pp. 5-13, Jan. 1989.

[30] A. van Dierendonck, P. Fenton, and T. Ford, "Theory and performance of narrow correlator spacing in a GPS receiver," J. Instit. Navigation, vol. 39, pp. 265-283, 1992.

[31] M. Wylie and J. Holtzmann, "The non-line of sight problem in mobile location estimation," in IEEE Int. Conf. Universal Personal Communications, 1996, pp. 827-831. 


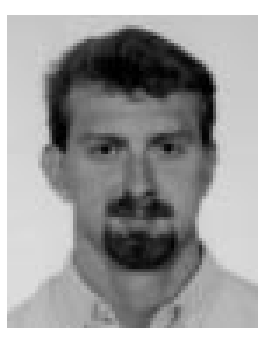

James Caffery, Jr. (S'91) was born in Alton, IL, in May 1970. He received the B.S.E.E. degree (summa cum laude) from Bradley University, Peoria, IL, in 1992 and the M.S. degree from the Georgia Institute of Technology, Atlanta, in 1993. He is currently working towards the Ph.D. degree at the Georgia Institute of Technology.

His research interests include CDMA systems, parameter estimation, and subscriber location in mobile cellular radio networks.
Gordon L. Stüber (S'81-M'86-SM'96) received the B.A.Sc. and Ph.D. degrees in electrical engineering from the University of Waterloo, Waterloo, Ont., Canada, in 1982 and 1986, respectively.

In 1986, he joined the School of Electrical and Computer Engineering, Georgia Institute of Technology, where he is currently a Professor. His research interests are in wireless communications and communication signal processing. He authored the textbook Principles of Mobile Communication (Boston, MA: Kluwer, 1996).

Dr. Stüber served as the Technical Program Chair for the 1996 IEEE Vehicular Technology Conference, Atlanta, GA, April 28-May 1, 1996. He is currently serving as the Technical Program Chair for the 1998 IEEE International Conference on Communications to be colocated with SUPERCOMM, Atlanta, GA, June 7-11, 1998. He served as an Editor for Spread Spectrum with the IEEE TRANSACTIONS ON COMMUNICATIONS from January 1993 to December 1997. 